\title{
L-arginine infusion decreases plasma total homocysteine concentrations through increased nitric oxide production and decreased oxidative status in Type II diabetic patients
}

\author{
M. Cassone Faldetta ${ }^{2}$, O. Laurenti ${ }^{3}$, G. Desideri ${ }^{1,2}$, M. C. Bravi², O. De Luca ${ }^{3}$, M. C. Marinucci², \\ G. De Mattia ${ }^{3}$, C. Ferri ${ }^{2}$ \\ ${ }^{1}$ University of L'Aquila, Department of Internal Medicine and Public Health, Via Vetoio 67100 - Blocco 11, Coppito, L'Aquila, \\ Italy \\ 2 Department of Internal Medicine and Public Health, University of L'Aquila, Italy \\ ${ }^{3}$ Department of Internal Medicine, University "La Sapienza”, Rome, Italy
}

\begin{abstract}
Aims/hypothesis. Hyperhomocysteinaemia increases cardiovascular risk in Type II (non-insulin-dependent) diabetes mellitus by augmenting oxidative stress and reducing nitric oxide availability. In vitro, nitric oxide decreases homocysteine by its conversion to the vasodilative and antioxidant compound S-nitrosohomocysteine. We investigated whether or not changes in nitric oxide availability decrease homocysteine concentrations in vivo.

Methods. The study group consisted of 20 normotensive, normolipidaemic, non-atherosclerotic Type II diabetic patients in good metabolic control (16 men, $51.2 \pm 1.4$ years) and 15 healthy subjects (12 men, $48.1 \pm 1.5$ years). Circulating concentrations of homocysteine, nitrite+nitrate and sulphydryl groups, a marker of oxidative stress, were assessed at baseline and then $5^{\prime}, 10^{\prime}, 30^{\prime}$ and $60^{\prime}$ after the intravenous infusion of either L-arginine ( $3 \mathrm{~g}$ in $10 \mathrm{ml}$ saline), the nitric oxide precursor, or vehicle according to a doubleblind cross-over randomized protocol.
\end{abstract}

Results. At baseline diabetic patients showed lower plasma sulphydryl group concentrations $(491.8 \pm 16.9$ vs $551.3 \pm 21.0 \mu \mathrm{mol} / 1, p<0.04)$ and nitrite+nitrate $(21.4 \pm 0.8$ vs $29.5 \pm 0.9 \mu \mathrm{mol} / 1, p<0.0001)$ and higher total homocysteine concentrations $(11.1 \pm 0.5$ vs $8.3 \pm 0.6 \mu \mathrm{mol} / 1, p<0.002)$ than the control subjects. After L-arginine infusion, blood pressure levels and total homocysteine concentrations $(p \leq 0.05)$ decreased (peak at $5^{\prime}$ and $30^{\prime}$, respectively) whereas nitric oxide and sulphydryl group concentrations $(p \leq 0.003)$ increased (peak at $10^{\prime}$ and $30^{\prime}$, respectively) in the patients and control subjects.

Conclusion/interpretation. Acute L-arginine infusion in both Type II diabetic patients and healthy subjects decreases plasma total homocysteine concentrations, counteract oxidative stress and increases the availability of nitric oxide. [Diabetologia (2002) 45:1120-1127]

Keywords Arginine, homocysteine, endothelium, diabetes, nitric oxide.
Homocysteine is a highly reactive thiol-containing amino acid derived from the conversion of methionine to cysteine [1]. In the presence of folate-, cobalamin-, and piridoxin-related compounds, newly-generated homocysteine is either transsulphurated to cysteine or remethilated to methionine. Therefore, mild to moder-

Received: 9 November 2001 / Revised: 4 March 2002

Published online: 22 June 2002

(C) Springer-Verlag 2002

Corresponding author: G. Desideri, MD, University of L'Aquila, Department of Internal Medicine and Public Health, Via Vetoio 67100 - Blocco 11, Coppito, L'Aquila, Italy. E-mail: giovambattista.desideri@cc.univaq.it. ate increases in plasma homocysteine concentrations can derive from inherited genetic defects that alter enzyme activities in the above transsulphuration and remethilation pathways [1], nutritional deficiencies of cobalamin, folate or piridoxine $[1,2]$ and reduced renal homocysteine excretion [3].

An increase in plasma total homocysteine concentrations has been described in subjects at increased cardiovascular risk, i.e. old people, smokers, men and women in the post-menopausal age [1, 2, 3, 4]. Accordingly, hyperhomocysteinaemia has also been reported in essential hypertension [5], renal failure [3], hyperlipidaemia [6], Type I (insulin-dependent) and Type II (noninsulin-dependent) diabetes mellitus [1,7]. 
The clinical relevance of hyperhomocysteinaemia in Type II diabetes is due to its association with the atherosclerotic disease [1]. In turn, the atherogenetic process has been suggested to be promoted by hyperhomocysteinaemia because of its detrimental influences on platelet aggregability $[1,8]$, coagulation cascade [9], vascular smooth muscle cell replication [10] and endothelial function $[1,11,12]$. With regard to endothelial function, homocysteine has been shown to exert direct cytotoxic effects on vascular endothelial cells $[1,13]$. In particular, homocysteine can reduce nitric oxide bioavailability by two main mechanisms. First, homocysteine can favour nitric oxide conversion to its pro-oxidant adduct peroxynitrite during homocysteine auto-oxidation [14]. Second, in the presence of homocysteine the vascular endothelial cell initially increases the production of nitric oxide with the consequent formation of s-nitrosohomocysteine [15], a homocysteine adduct which preserves the bioactivity of nitric oxide with vasodilatory and antiplatelet effects $[16,17]$. This effect of homocysteine is caused by changes in the oxidative status and inducible nitric oxide synthase expression and strongly limits homocysteine-mediated vascular endothelial damage [17]. However, prolonged exposure to high homocysteine concentrations progressively exhausts nitric oxide bioavailability and thereby impairs endothelium-dependent vasorelaxation $[17,18]$. In turn, the same homocysteine-related decrement in nitric oxide bioavailability further reduces homocysteine conversion to s-nitrosohomocysteine and favours the development of endothelial damage, vasoconstriction and platelet aggregation.

An improved nitric oxide formation by the vascular endothelium is obviously expected to ameliorate homocysteine conversion to S-nitrosohomocysteine and to reduce oxidative stress. Accordingly, infusion of the nitric oxide precursor L-arginine has been reported to restore nitric oxide bioavailability and improve endothelium-dependent vasorelaxation [19] in Type II but not in Type I diabetic patients [20]. However, at present studies on the behaviour of plasma total homocysteine and oxidative status in diabetic patients infused with L-arginine are not available. To address this topic, we intravenously administrated L-arginine to uncomplicated Type II diabetic patients in good metabolic control and without additional risk factors. Thus, plasma nitrite+nitrate, total homocysteine and sulphydryl group concentrations, i.e. a well recognised marker of oxidative status in vivo [21], were measured in the same patients.

\section{Subjects and methods}

Patients. The study protocol was approved by the local Ethics Committee. Type II diabetic patients (16 men and 4 women, mean age 51.2 \pm 1.4 years) were selected among consecutive Type II diabetic outpatients who were managed by our Diabe- tes Outpatients Clinic since their first diagnosis of diabetes and were seen for scheduled controls during the year 2000. In all patients, Type II diabetes was diagnosed according to the criteria of the American Diabetes Association [22]. Exclusion criteria were: Type I diabetes as well as any diabetes other than Type II diabetes [22], hypertension (sitting blood pressure levels $\geq 140 / 90 \mathrm{mmHg}$ ), serum cholesterol and triglyceride concentrations greater than or equal to $5.2 \mathrm{mmol} / \mathrm{l}$ and $1.7 \mathrm{mmol} / \mathrm{l}$, respectively, proliferative retinopathy, personal history of coronary or cerebrovascular diseases, abnormalities at the resting 12-lead electrocardiogram, claudicatio intermittens, severe peripheral neuropathy, cigarette smoking, alcohol consumption of more than $10 \mathrm{~g}$ a day, positive microalbuminuria (as evaluated from three consecutive morning urine spots), ultrasound evidence of atherosclerotic lesions of the neck and limb vessels including a carotid intima-media thickness of $1.1 \mathrm{~mm}$ or more, concomitant diseases, treatment with any kind of drug including vitamins, antihypertentive, lipid-lowering or antiplatelet agents, etc. All patients were in good metabolic control achieved by their diet and glibenclamide treatments.

l-arginine infusion. After informed consent, each patient was interviewed by the experienced dieticians who designed their individual diet at the time of their first diagnosis of Type II diabetes and filled a nutritional diary. Then, the individual diet was adjusted for each patient, without changing total calories or diet components, but excluding foods containing antioxidants and limiting nitrite and nitrate intake ( $\leq 180 \mu \mathrm{mol} /$ day $)$ [23]. Patient compliance with the assigned diet was ensured by consulting weekly the daily dietary diaries that all patients were asked to fill upon participating in the study. After 4 weeks on this diet, experiments were carried out in our Outpatient Clinic after an overnight fast. Briefly, at 8:00 hours an intravenous lockable device was located in the left arm to collect venous blood samples and administrate either L-arginine (Damor Pharmaceuticals, Naples, Italy) diluted in $10 \mathrm{ml}$ isotonic saline, given at a dose of $3 \mathrm{~g}$ over a period of $60 \mathrm{~s}$, or placebo (10 $\mathrm{ml}$ isotonic saline) with the patient recumbent in supine position and relaxed, according to a randomised, doubleblind, cross-over protocol. An automated blood pressure device (Omega 1400, Spacelab, Redmond, Wash., USA ) was applied to the contralateral arm to monitor systolic and diastolic blood pressure levels and heart rate at 5 min intervals. Then, after $30 \mathrm{~min}$ at rest without leaving the supine position, blood was collected for measuring fasting glucose, serum total cholesterol, high density lipoprotein cholesterol, triglycerides, glycated haemoglobin, and plasma insulin, sulphydryl group, total plasma homocysteine and nitrite+nitrate concentrations, i.e. a well-recognised marker of nitric oxide production in vivo [24].

After basal blood samplings, either L-arginine or placebo were randomly infused. Then, blood samples were collected again after a period of 5, 10, 30 and 60 minutes from L-arginine or saline administration to determine serum glucose and plasma insulin, total homocysteine, sulphydryl group and nitrite+nitrate concentrations.

The double-blind, cross-over, randomised procedure assigned 11 patients to receive first the placebo and then the L-arginine administration and 9 patients in the reverse order. A one week interval occurred between the two infusions, during which the assigned diet was not discontinued. On both infusion days, the daily glibenclamide dose was not administrated.

Control group. The above protocol was also applied for studying 15 healthy volunteers (12 men and 3 women, mean age $48.1 \pm 1.5$ years) recruited among the personnel of our Institu- 
tions. Selection criteria for the control subjects were as for Type II diabetic patients, but an impaired glucose metabolism [22] was considered as an additional exclusion criterion. The double-blind, cross-over, randomised procedure assigned seven subjects to receive firstly the placebo and then the L-arginine administration and eight subjects in the reverse order. One week interval occurred between the two infusions.

Both Type II diabetic patients and the control subjects gave their consent after the nature of the procedure was explained.

Infusion of l-arginine + octreotide. L-arginine can modestly increase insulin secretion $[24,25]$. To avoid the bias of L-arginine-related changes in plasma insulin concentrations after the above infusion studies, Type II diabetic patients and control subjects were re-infused with L-arginine but after endogenous insulin secretion was suppressed. For this purpose, L-arginine was infused (again, $3 \mathrm{~g}$ diluted in $10 \mathrm{ml}$ isotonic saline over a period of $60 \mathrm{~s}$ ). After $30 \mathrm{~min}$ an intravenous administration of the somatostatin analogue octreotide $(0.1 \mathrm{mg}$ bolus followed by $0.2 \mathrm{mg}$ for $90 \mathrm{~min}$ ) was started. As in the previous studies, blood samplings for plasma total homocysteine, nitrite+nitrate and sulphydryl groups concentrations were made after $0,5,10$, 30 and $60 \mathrm{~min}$ from the L-arginine infusion.

Biochemical measurements. Serum glucose, total cholesterol, high density lipoprotein cholesterol and triglycerides were assessed by enzymatic methods (Boehringer Mannheim, Mannheim, Germany). Low density lipoprotein cholesterol was calculated by the well-known Friedewald formula. Plasma insulin was assessed by radioimmunoassay (Biochem Immuno Systems, Italia).

Glycated haemoglobin assay was carried out by immunoturbidimetric assay based on the principle of inhibition of latex agglutination (DCA 2000, Bayer Diagnostics, Milan, Italy).

Plasma L-arginine concentrations were assessed using high performance liquid chromatography with fluorescence detection [26].

Plasma sulphydryl groups were measured with a spectrophotometric according to a method described previously [27].

Since homocysteine circulates in both the reduced and oxidized forms, homocysteine was evaluated as total homocysteine, i.e. oxidized + reduced according to widely accepted recommendations [1]. For this purpose, blood was collected in pre-chilled EDTA containing tubes and immediately centrifuged at $4^{\circ} \mathrm{C}$, then separated and stored at $-20^{\circ} \mathrm{C}$ until the assay. The well recognised method used was the fluorescence polarisation immunoassay by the IMX System (Abbott Diagnostics, Il., USA) [28]. To completely exclude the possible interference of L-arginine on plasma total homocysteine concentration assessment, plasma total hymocysteine assay was repeated in some randomised plasma samples $(n=10)$ from either the patients or the control subjects after different amounts of L-arginine were added. The data showed that L-arginine addition did not interfere with plasma total homocysteine assay.

Nitric oxide was evaluated by the well recognised method based on the indirect measurements of the nitric oxide-based by-products nitrite and nitrate [24] for which blood samples were treated as for homocysteine. Then nitric oxide concentrations were evaluated with measurements of nitrite plus nitrate by colorimetric detection of nitrite after conversion of all the sample nitrate into nitrite (Assay Design, Ann Arbor, Mich., USA).

Statistical analysis. Data collection and storage were made by a common hardware system and the SPSS package (Chicago, Ill., USA). Comparisons within the groups were made by paired Student's $t$ tests. To compare the responses to L-arginine the analysis of variance with appropriate adjustment for multiple comparisons was used. Relation between the dependent variables plasma nitrite+nitrate; total homocysteine and sulphydryl group concentrations and changes in plasma nitrite+nitrate, total homocysteine and sulphydryl concentrations; and the independent variables, age, sex, diabetes duration (for Type II diabetic patients only), total cholesterol, cholesterol subfractions, triglycerides, glycated haemoglobin, glucose and insulin was evaluated by univariate and then multivariate analysis. Unless otherwise stated, data are expressed as mean \pm SE. Significances were given for $p$ values of less than 0.05 .

\section{Results}

Baseline data. There were no significant differences in age, sex distribution, blood pressure, heart rate, serum total cholesterol and cholesterol subfractions, triglyceride, insulin and L-arginine concentrations and urinary albumin excretion between the two groups (Table 1). As expected, fasting glucose concentrations and glycated haemoglobin concentrations were higher in Type II diabetic patients than in the control subjects. In addition, the diabetic patients had increased plasma total homocysteine concentrations and decreased plasma sulphydryl group concentrations compared with the control subjects. Plasma nitrite+nitrate concentrations were also lower in Type II diabetic patients than in the control subjects. There were no correlations between the dependent variables baseline plasma total homocysteine and sulphydryl group concentrations and the other variables used as independent ones.

Table 1. Clinical and fasting biochemical features of Type II diabetic patients $(n=20)$ and healthy volunteers $(n=15)$. Data are shown as means $\pm \mathrm{SE}$

\begin{tabular}{lcc}
\hline Variable & Patients & $\begin{array}{c}\text { Control } \\
\text { subjects }\end{array}$ \\
\hline Age (years) & $51.2 \pm 1.4$ & $48.1 \pm 1.5$ \\
Sex (men/women) & $16 / 4$ & $12 / 3$ \\
Disease duration (years) & $4.8 \pm 0.9$ & - \\
Systolic BP (mmHg) & $129.5 \pm 2.4$ & $126.1 \pm 1.8$ \\
Diastolic BP (mmHg) & $79.4 \pm 1.4$ & $78.4 \pm 0.7$ \\
Heart rate (beats/min) & $72.4 \pm 1.9$ & $68.9 \pm 1.2$ \\
Plasma glucose $(\mathrm{mmol} / \mathrm{l})$ & $6.6 \pm 0.2^{\mathrm{a}}$ & $4.9 \pm 0.1$ \\
Plasma insulin $(\mathrm{pmol} / \mathrm{l})$ & $94.1 \pm 3.4$ & $84.5 \pm 4.5$ \\
Glycated haemoglobin $(\%)$ & $5.5 \pm 0.2^{\mathrm{b}}$ & $4.6 \pm 0.2$ \\
Serum total cholesterol $(\mathrm{mmol} / \mathrm{l})$ & $4.2 \pm 0.1$ & $3.9 \pm 0.1$ \\
Serum HDL (mmol/l) & $1.1 \pm 0.05$ & $1.1 \pm 0.05$ \\
Serum LDL (mmol/l) & $2.9 \pm 0.1$ & $2.6 \pm 0.1$ \\
Serum triglycerides $(\mathrm{mmol} / \mathrm{l})$ & $1.2 \pm 0.05$ & $1.2 \pm 0.02$ \\
Plasma total homocysteine $(\mu \mathrm{mol} / \mathrm{l})$ & $11.1 \pm 0.5^{\mathrm{b}}$ & $8.3 \pm 0.6$ \\
Plasma sulphydryl groups $(\mu \mathrm{mol} / \mathrm{l})$ & $491.8 \pm 16.9^{\mathrm{c}}$ & $551.3 \pm 21.0$ \\
Plasma nitrite+nitrate $(\mu \mathrm{mol} / \mathrm{l})$ & $21.4 \pm 0.8^{\mathrm{a}}$ & $29.5 \pm 0.9$ \\
L-arginine $(\mu \mathrm{mol} / \mathrm{l})$ & $75.2 \pm 2.4$ & $68.4 \pm 3.5$ \\
Urinary albumin excretion $(\mu \mathrm{g} / \mathrm{min})$ & $9.8 \pm 1.0$ & $8.8 \pm 0.8$ \\
\hline
\end{tabular}

${ }^{\mathrm{a}} p<0.0001 \quad{ }^{\mathrm{b}} p<0.002 \quad{ }^{\mathrm{c}} p<0.04$


Table 2. Serum glucose, plasma insulin concentrations and blood pressure during L-arginine infusion in 20 Type II diabetic patients and 15 healthy volunteers

\begin{tabular}{|c|c|c|c|c|c|}
\hline Patients & $0^{\prime}$ & $5^{\prime}$ & $10^{\prime}$ & $30^{\prime}$ & $60^{\prime}$ \\
\hline L-arginine $(\mu \mathrm{mol} / \mathrm{l})$ & $74 \pm 2$ & $9168.1 \pm 171^{a}$ & $4218 \pm 144^{a}$ & $2550 \pm 122^{\mathrm{a}}$ & $2053 \pm 55$ a \\
\hline Glucose (mmol/l) & $6.7 \pm 0.2$ & $6.2 \pm 0.2^{b}$ & $5.9 \pm 0.1^{\mathrm{c}}$ & $6.4 \pm 0.2$ & $6.6 \pm 0.2$ \\
\hline Insulin $(\mathrm{pmol} / \mathrm{l})$ & $97.6 \pm 3.1$ & $112.2 \pm 3.0^{\mathrm{c}}$ & $107.7 \pm 3.4^{b}$ & $101.6 \pm 3.0$ & $100.0 \pm 3.3$ \\
\hline Diastolic BP (mmHg) & $80.1 \pm 1.1$ & $75.0 \pm 1.0^{\mathrm{c}}$ & $77.1 \pm 1.0^{\mathrm{b}}$ & $80.6 \pm 1.0$ & $81.0 \pm 1.2$ \\
\hline Control subjects & $0^{\prime}$ & $5^{\prime}$ & $10^{\prime}$ & $30^{\prime}$ & $60^{\prime}$ \\
\hline Glucose $(\mathrm{mmol} / \mathrm{l})$ & $4.5 \pm 0.1$ & $4.2 \pm 0.1^{b}$ & $4.2 \pm 0.1^{b}$ & $4.6 \pm 0.1$ & $4.6 \pm 0.1$ \\
\hline Insulin $(\mathrm{pmol} / \mathrm{l})$ & $85.9 \pm 4.4$ & $104.7 \pm 3.8^{\mathrm{e}}$ & $99.5 \pm 4.3^{b}$ & $91.3 \pm 4.1$ & $86.9 \pm 4.2$ \\
\hline Systolic BP (mmHg) & $126.4 \pm 1.7$ & $118.5 \pm 1.8^{e}$ & $121.2 \pm 1.6^{b}$ & $124.4 \pm 1.8$ & $125.3 \pm 1.6$ \\
\hline Diastolic BP (mmHg) & $78.3 \pm 0.6$ & $74.8 \pm 0.8^{c}$ & $76.5 \pm 0.5^{b}$ & $78.7 \pm 0.6$ & $79.1 \pm 0.7$ \\
\hline
\end{tabular}

${ }^{\mathrm{a}} p<0.0001 \quad{ }^{\mathrm{b}} p<0.04 \quad{ }^{\mathrm{c}} p<0.002 \quad{ }^{\mathrm{d}} p<0.02 \quad{ }^{\mathrm{e}} p<0.004$

Data are given as means \pm SE. Significances are given vs baseline

Infusion data. After L-arginine infusion endogenous plasma concentrations reached a maximum at $5 \mathrm{~min}$ and then rapidly declined in the Type II diabetic patients and the control subjects (Table 2). Plasma L-arginine concentrations were still higher than the baseline after $60 \mathrm{~min}$ (Table 2). Blood pressure changed with L-arginine infusion in both groups with a peak at $5 \mathrm{~min}$. This effect was still significant ( $p<0.04$ or less) after $10 \mathrm{~min}$ but it disappeared after 30 min. L-arginine infusion also induced changes ( $p<0.04$ or less) in plasma insulin concentrations in the Type II diabetic patients and the control subjects (Table 2). Increments in plasma insulin concentrations were transient, with a rapid return to baseline concentrations after $30 \mathrm{~min}$ from the L-arginine administration. In contrast to insulin concentrations and blood pressure, serum glucose concentrations very modestly decreased during L-arginine infusion in the Type II diabetic patients as well as in the control subjects (Table 2). As expected, placebo infusion did not induce changes in the above variables in both groups.

L-arginine administration decreased the circulating concentration plasma total homocysteine in Type II diabetic patients and, to a lesser degree, in healthy subjects (Fig. 1). Total plasma homocysteine concentrations were lower than at baseline after 10 and $30 \mathrm{~min}$ from the infusion and returned to pre-infusion values after $60 \mathrm{~min}$. Statistical analysis did not show differences in plasma total homocysteine responses to L-arginine between the Type II diabetic patients and the control subjects. An inverse correlation between changes in plasma homocysteine and L-arginine concentrations was observed in the Type II diabetic patients $(r=-0.446, p=0.049)$ and the control subjects $(r=-0.568, p=0.027)$ at time $30 \mathrm{~min}$.

L-arginine administration increased plasma nitrite+nitrate concentrations in both Type II diabetic patients and control subjects (Fig. 2). The time course
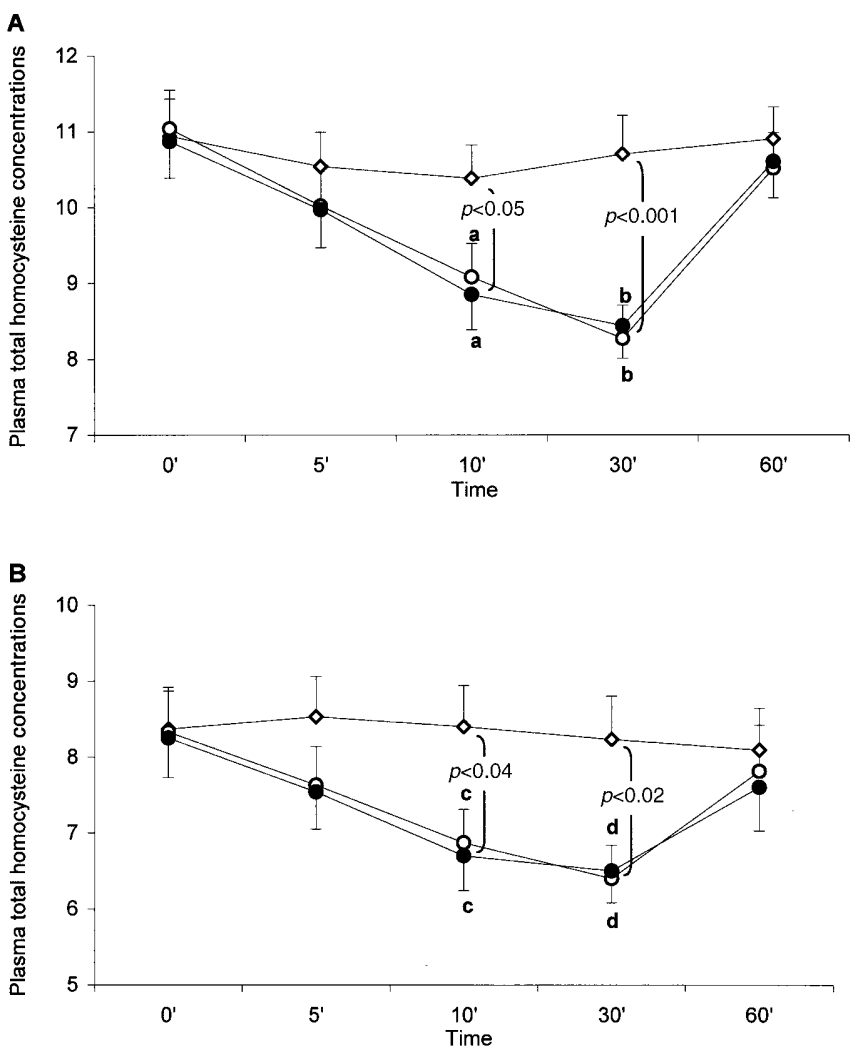

Fig. 1A, B. Plasma total homocysteine concentrations ( $\mu \mathrm{mol} / \mathrm{l})$ in 20 Type II diabetic patients (A) and 15 control subjects (B) during the intravenous infusion of either saline (white rhombuses) or L-arginine (white circles) or L-arginine + octreotide (black circles). Data are shown as means \pm SE. ${ }^{\mathrm{a}} p<0.007$, ${ }^{\mathrm{b}} p<0.0002,{ }^{\mathrm{c}} p<0.05,{ }^{\mathrm{d}} p<0.01$ vs baseline

of the changes in plasma nitric oxide metabolite concentrations was mirrored in the pattern of changes in plasma L-arginine concentrations. In particular, nitrite+nitrate concentrations increased after 5 and $10 \mathrm{~min}$, with a return to baseline after $30 \mathrm{~min}$. Signifi- 

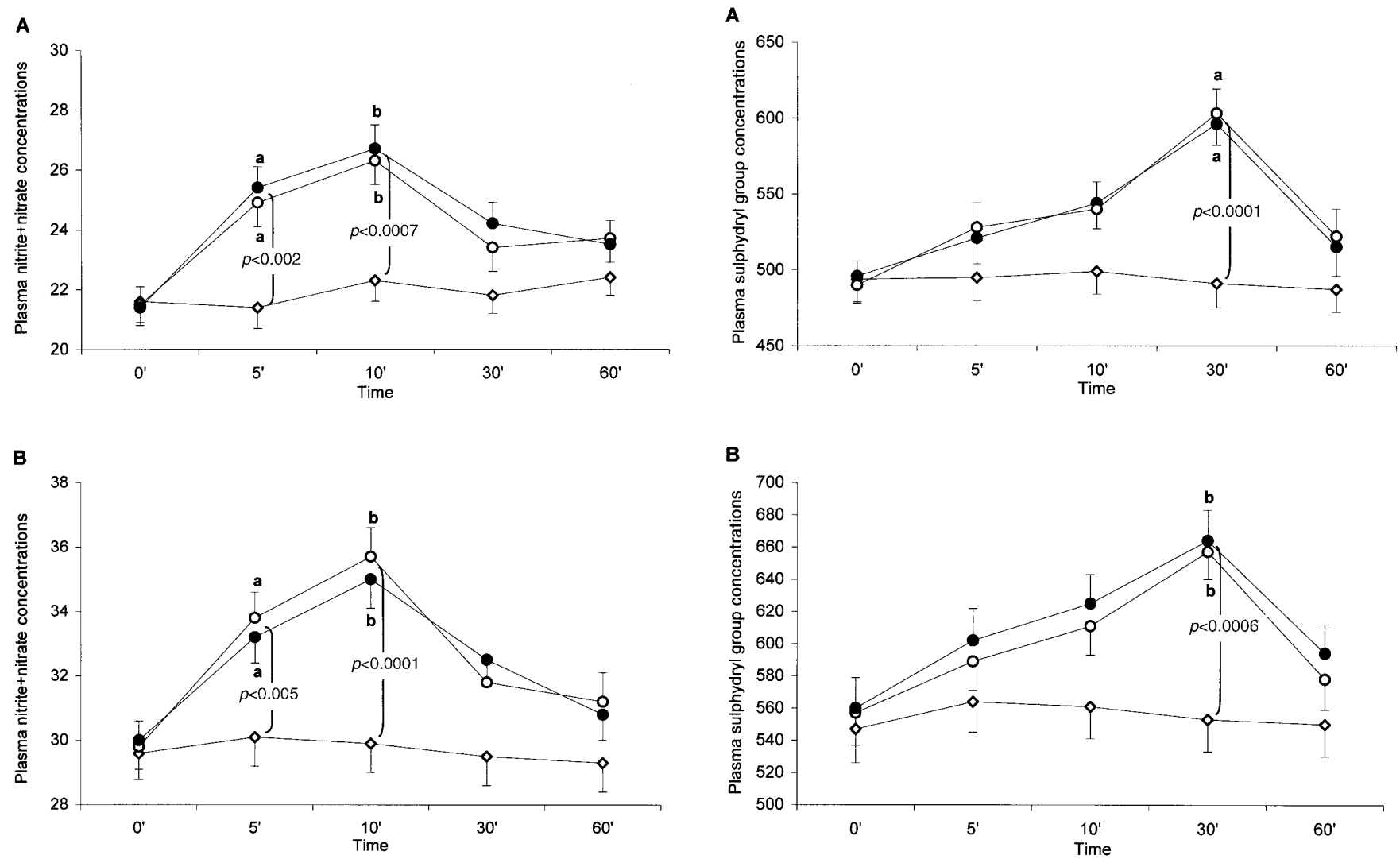

Fig. 2A, B. Plasma nitrite+nitrate concentrations ( $\mu \mathrm{mol} / \mathrm{l})$ in 20 Type II diabetic patients (A) and 15 control subjects $(\mathbf{B})$ during the intravenous infusion of either saline (white rhombuses) or L-arginine (white circles) or L-arginine + octreotide (black circles). Data are shown as means \pm SE. ${ }^{\mathrm{a}} p<0.003,{ }^{\mathrm{b}} p<0.0001$ vs baseline

cant increments $(p<0.0009$ or less) in plasma sulphydryl group concentrations were observed after $30 \mathrm{~min}$ (Fig. 3). Plasma sulphydryl groups returned to baseline after $60 \mathrm{~min}$ from the infusion. As with the total plasma homocysteine, statistical analysis failed to show differences in plasma sulphydryl groups and nitrite+nitrate responses to L-arginine between the Type II diabetic patients and the control subjects.

Statistical analysis showed no order effect dependent on whether L-arginine was infused before placebo and vice versa in the patient and control groups.

As expected, when diabetic patients and control subjects were re-infused with L-arginine after the secretion of endogenous insulin was suppressed by the somatostatin analogue octreotide, circulating insulin concentrations decreased $(p<0.0001)$ within $30 \mathrm{~min}$ of octreotide infusion. Compared with L-arginine alone, L-arginine + ocreotide infusion induced identical changes in plasma total homocysteine, nitrite+ nitrate and sulphydryl groups responses (Figs. 1, 2 and 3 ).
Fig. 3A, B. Plasma sulphydryl group $(\mu \mathrm{mol} / \mathrm{l})$ concentrations in 20 Type II diabetic patients (A) and 15 control subjects $(\mathbf{B})$ during the intravenous infusion of either saline (white rhombuses) or L-arginine (white circles) or L-arginine + octreotide (black circles). Data are shown as means $\pm \mathrm{SE} .{ }^{\mathrm{a}} p<0.0001$, $\mathrm{b}_{p}<0.0009$ vs baseline

\section{Discussion}

Our study shows that plasma total homocysteine concentrations increased, while plasma sulphydryl group and nitrite+nitrate concentrations decreased, in very carefully selected Type II diabetic patients in good metabolic control and without disturbances of the lipid metabolism, high blood pressure, and target organ damage. Our findings suggest that Type II diabetes in itself, i.e. regardless to the presence or the absence of chronic hyperglycaemia and macrovascular complications, induces endothelial dysfunction, being accompanied by (i) hyperhomocysteinaemia, (ii) impaired nitric oxide production, and (iii) increased oxidative stress. The most important outcome was that all of these abnormalities are L-arginine-inhibitable, at least in our experimental conditions.

The possible pro-atherogenic role of homocysteine has been extensively studied in diabetic and non-diabetic subjects [1]. In particular, plasma total homocysteine concentrations have been reported as correlated with blood pressure behaviour [29], the presence of vascular damage [30] and the rate of urinary albumin 
excretion in Type II diabetic patients [29, 30]. Similarly, increased plasma total homocysteine concentrations directly correlated with urinary albumin excretion have been recently described in uncomplicated Type II diabetic patients in good metabolic control [31]. Thus, the increased plasma total homocysteine concentration that we found in uncomplicated Type II diabetic patients in good metabolic control is not surprising. However, the lack of correlations between urinary albumin and plasma total homocysteine concentrations in our patients simply reflects the exclusion of Type II diabetic patients with early nephropathy from our study.

Type II diabetic patients also showed lower plasma nitrite+nitrate concentrations than the control subjects. These data agree with a number of studies showing an impaired endothelium-dependent vasorelaxation in different vascular districts of Type II diabetic patients [24]. In this context, decreased plasma concentrations of the second messenger of nitric oxide, i.e. cyclic guanosine monophosphate, have been recently described in Type II diabetic patients [32]. Nevertheless, in contrast with our data, nitrite+nitrate concentrations were not reduced in the same diabetic patients [32]. Discrepancies with our data are likely due to uncontrolled nitrite and nitrate intake with diet [23] in the previous quoted study [32]. As far as mechanisms leading to reduced nitric oxide availability in our diabetic patients, it is of interest that Type II diabetic patients show sulphydryl group concentrations than the control subjects. Thus, it is likely that homocysteineinduced oxidative stress [14] might lead to enhanced nitric oxide degradation by favouring its conversion to peroxynitrite $[1,14]$. Moreover, excess homocysteine is known to affect nitric oxide availability also by impairing nitric oxide synthase pathway [33] and interacting with nitric oxide to form the vasodilative adduct S-nitrosohomocysteine and the consequent exhaustion of the endothelial cell capability to produce a relevant amount of nitric oxide $[1,15,17]$.

The principal outcome from our study is the reduction of plasma total homocysteine concentrations observed after L-arginine infusion in both Type II diabetic patients and control subjects. Interestingly, in both groups changes in plasma total homocysteine concentrations were inversely related to changes in L-arginine concentrations induced by L-arginine infusion. This finding shows that L-arginine infusion is able to reduce homocysteine concentrations in both Type II diabetic patients and healthy subjects. Plasma homocysteine has not been assessed in previous studies showing the positive effects of L-arginine on insulin-mediated [34] and endothelium-dependent vasorelaxation [24] as well as on plasma nitrite+ nitrate concentrations in diabetic patients [35]. Thus, L-arginine-mediated decrement in plasma homocysteine concentrations seems to represent a newly discovered, additional benefit which could derive from arginine supplementation in diabetic patients [24].
In terms of mechanics, an increase $(p<0.003$ or less) in nitric oxide metabolite concentrations was observed after L-arginine infusion in Type II diabetic patients and by nearly equivalent degrees in the control subjects. These data are in substantial agreement with previous findings in Type II diabetic patients [24] as well as in healthy subjects [24] and suggest an improvement in nitric oxide availability. Accordingly changes in nitric oxide metabolite concentration and blood pressure simultaneously achieved the maximum peak between both groups. The time course of nitric oxide metabolite concentrations was mirrored in the pattern of changes in plasma total homocysteine concentrations. Thus, it is appealing to speculate that the L-arginine-related decrement in plasma homocysteine concentration observed in Type II diabetic patients and control subjects was due to an acute increment in nitric oxide availability. In this context it is interesting to observe that L-arginine infusion reduced oxidative stress, as suggested by the increment in plasma sulphydryl group concentrations, in Type II diabetic patients and control subjects. Thus, we could speculate that increased nitric oxide availability due to L-arginine infusion induces a decrement in homocysteine concentrations which in turn counteracts oxidative stress. On this basis, plasma-sulphydryl group-changes achieved the maximum peak after a plasma homocysteine decrement was achieved. Since the above findings were identical in Type II diabetic patients and healthy subjects it is evident that these effects are physiologic, i.e. independent of diabetes. However, our Type II diabetic patients were in good metabolic control and without ultrasound-detectable vascular lesions. Since hyperglycaemia markedly contributes to the progression of endothelial dysfunction and impairs inducible nitric oxide synthesis [24] as well as increase oxidative stress, we cannot transpose our findings to Type II diabetic patients in poor metabolic control.

Endogenous insulin release during L-arginine infusion could be involved in L-arginine-related reduction of plasma total homocysteine. Indeed, in agreement with other data $[24,25]$ we confirm that L-arginine increases plasma insulin concentrations in both Type II diabetic patients and control subjects. Therefore, since insulin is known to stimulate the endothelial production of both endothelin-1 [36] and nitric oxide [36, 37], the observed L-arginine-related changes in plasma total homocysteine concentrations could have been due, at least in part, to transient hyperinsulinaemia. In contrast to this hypothesis, simultaneous infusion of octreotide, i.e. a somatostatin analogue which is known to block endogenous insulin secretion without altering haemodynamic and other metabolic variables $[38,39]$, did not modify L-arginine effects on plasma total homocysteine, sulphydryl groups, and nitrite+ nitrate concentrations in Type II diabetic patients and 
control subjects. Thus, the L-arginine-related reduction of plasma total homocysteine concentrations was not insulin-mediated.

In conclusion, our study shows that acute L-arginine infusion in both Type II diabetic patients and healthy subjects increases nitric oxide availability, counteracts oxidative stress and decreases plasma total homocysteine concentrations.

\section{References}

1. Guba SC, Fink LM, Fonseca V (1996) Hyperhomocysteinemia. An emerging risk factor for thromboembolic and cardiovascular disease. Am J Clin Pathol 105:709-722

2. Allen RH, Stabler SP, Savage DG, Lindembaum J (1994) Metabolic abnormalities in cobalamin (vitamin B-12) and folate deficiency. FASEB J 7:1344-1353

3. Arnadottir M, Hultberg B, Nilsson-Ehle P, Thysell H (1996) The effect of reduced glomerular filtration rate on plasma total homocysteine concentration. Scand J Clin Lab Invest 56:41-46

4. Rasmussen K, Moller M, Lyngbak M, Pederse AM, Dybkjaer L (1996) Age and gender specific reference intervals for total homocysteine and methylmalonic acid in plasma before and after vitamin supplementation. Clin Chem 42:630-636

5. Cassone-Faldetta M, Laurenti O, De Luca O et al. (2000) Elevated circulating homcysteine levels in uncomplicated essential hypertensives with and without type 2 diabetes mellitus. High Blood Press 9:141-145

6. Glueck CJ, Shaw P, Lang JE, Tracy T, Sieve-Smith L, Wang Y (1995) Evidence that homocysteine is an independent risk factor for atherosclerosis in hyperlipidemic patients. Am J Cardiol 75:132-136

7. Hoogeveen EK, Kostense PJ, Beck PJ et al. (1998) Hyperhomocysteinemia is associated with an increased risk of cardiovascular disease, especially in non-insulin-dependent diabetes mellitus: a population-based study. Arterioscler Thromb Vasc Biol 18:133-138

8. Di Minno G, Davì G, Margaglione M (1993) Abnormally high thromboxane byosynthesis in homozygous homocystinuria: evidence for platelet involvement and probucol-sensitive mechanism. J Clin Invest 92:1400-1406

9. Harpel PC, Chang VT, Borth W (1992) Homocysteine and other sulphydryl compounds enhance the binding of lipoprotein (a) to fibrin: a potential link between thrombosis, atherogenesis and sulphydryl compound metabolism. Proc Natl Acad Sci USA 89:10193-10197

10. Tsai JC, Perrella MA, Yoshizumi M et al. (1994) Promotion of vascular smooth muscle cell growth by homocysteine: a link to atherosclerosis. Proc Natl Acad Sci USA 91:6369-6373

11. Stamler JS, Loscalzo J (1992) Endothelium-derived relaxing factor modulates the atherothrombogenic effects of homocysteine. J Cardiovasc Pharmacol 20:S202-S204

12. Tawakol A, Tornbjorn O, Gerhard M, WU JT, Creager MA (1997) Hyperhomocyst(e)inemia is associated with impaired endothelium-dependent vasodilation in humans. Circulation 95:1119-1121

13. Wall RT, Horlan JM, Harker LA, Striker GE (1980) Homocyst(e)ine induced endothelin injury in vitro: a model for the study of vascular injury. Thromb Res 18:113-121

14. Loscalzo J (1996) The oxidation of hyperhomocysteinemia. J Clin Invest 98:5-8
15. Upchurch GR Jr, Welch GN, Fabian AJ, Pigazzi A, Keaney JF Jr, Loscalzo J (1997) Stimulation of endothelial nitric oxide production by homocyst(e)ine. Atherosclerosis 132: 177-185

16. Ikeda U, Ikeda M, Minota S, Shimada K (1999) Homocysteine increases nitric oxide synthesis in cytokine-stimulated vascular smooth cells. Circulation 99:1230-1235

17. Stamler JS, Osborne JA, Jaraki O et al. (1993): Adverse vascular effects of homocysteine are modulated by endothelium-derived relaxing factor and related oxides of nitrogen. J Clin Invest 91:303-318

18. Chao CH, Kuo TL, Lee YT (2000) Effects of methionineinduced hyperhomocysteinemia on endothelium-dependent vasodilation and oxidative status in healthy adults. Circulation 101:485-490

19. Thorne S, Mullen MJ, Clarkson P, Donald AE, Deanfield JE (1998) Early endothelial dysfunction in adults at risk from atherosclerosis: different respose to L-arginine. J Am Coll Cardiol 32:110-116

20. Giugliano D, Marfella D, Terrazzo G et al. (1997) L-arginine for testing endothelium-dependent vascular functions in health and disease. Am J Physiol 273:E606-E612

21. Telci A, Cakatay U, Salman S, Staman I, Sivas A (2000) Oxidative protein damage in early stage of type 1 diabetic patients. Diabetes Res Clin Pract 50:213-223

22. American Diabetes Association (1998) Report of the Expert Committee on the diagnosis and classification of diabetes mellitus (Committee Report). Diabetes Care 21:S5S19

23. Granger DL, Taintor RR, Boockwar KS, Hibbs JB Jr (1996) Measurement of nitrate and nitrite in biological samples using nitrate reductase and Griess Reaction. Methods Enzymol 268:142-151

24. Pieper GM (1998) Review of alterations in endothelial nitric oxide production in diabetes. Protective role of arginine on endothelial dysfunction. Hypertension 31:10471060

25. Giugliano D, Marfella R, Verrazzo G et al. (1997) The vascular effects of L-arginine in humans. The role of endogenous insulin. J Clin Invest 99:433-438

26. Golapakrishnan V, Burton PJ, Blasche TF (1996) Highperformance liquid chromatographic assay for the quantitation of L-arginie in human plasma. Anal Chem 68:35203523

27. Ellmann GL, Boyne AF (1972) A methodology for analysis of tissue sulphydryl components. Anal Biochem 46: 639-653

28. Shipchandler MT, Moore EG (1995) Rapid, fully automated measurement of plasma homocyst(e)ine with the Abbott Imx Analyser. Clin Chem 41:991-994

29. Fiorina P, Lanfredini M, Montanari A et al. (1998) Plasma homocysteine and folate are related to arterial pressure in type 2 diabetes. Am J Hypertens 11:1110-1117

30. Lanfredini M, Fiorina P, Peca MG et al. (1998) Fasting and post-methionine load homocysteine values are correlated with microalbuminuria and could contribute to worsening vascular damage in non-insulin-dependent diabetes mellitus subjects. Metabolism 47:915-921

31. Chico A, Perez A, Cordoba A et al. (1998) Plasma homocysteine is related to albumin excretion rate in patients with diabetes mellitus: a new link between diabetic nephropathy and cardiovascular disease? Diabetologia 41: 684-693

32. Aydin A, Orhan H, Sayal A, Ozata M, Sahin G, Isimer A (2001) Oxidative stress and nitric oxide related parameters in type II diabetes mellitus: effects of glycemic control. Clin Biochem 34:65-67 
33. Sthhlinger MC, Tsao PS, Her JH et al. (2001) Homocysteine impairs the nitric oxide synthase pathway. Role of asymmetric dimethylarginine. Circulation 104:2569-2575

34. Wascher TC, Graier WF, Hussain MA, Bahadori B, Wallner S, Toplak H (1997) Effect of low dose L-arginine on insulin-mediated vasodilation and insulin sensitivity. Eur J Clin Invest 27:690-695

35. Das UN, Mohan IK, Kumar KV, Kumar GS, Sekhar CC (1992) Beneficial effect of L-arginine in non-insulindependent diabetes mellitus: a potential role for nitric oxide. Med Sci Res 255:721-723

36. Desideri G, Ferri C, Bellini C, De Mattia G, Santucci A (1997) Effects of ACE inhibition on spontaneous and insulin-stimulated endothelin-1 secretion. Diabetes 46:81-86
37. Steinberg HO, Brechtel G, Johnson A, Fineberg N, Baron AD (1994) Insulin-mediated skeletal muscle vasodilation is nitric oxide dependent. A novel action of insulin to increase nitric oxide release. J Clin Invest 94:1172-1179

38. Ferri C, De Mattia G, Bellini et al. (1993) Octreotide, a somatostatin analog, reduces insulin secretion and increase renal $\mathrm{Na}+$ excretion in lean essential hypertensive patients. Am J Hypertens 6:276-281

39. Gordon PH, Comi RJ, Matom PM (1989) Somatostatin and somatostatin analogue SMS-201-995 in treatment of hormone secretin tumours of the pituitary and gastrointestinal tract and non-neoplastic diseases of the gut. Ann Intern Med 110:35-50 